Radioactive phosphorus, ${ }^{32} \mathrm{P}$ is administered intravenously about 15 hour before the operation. Counting ratios of brain tumors are compared with norma brain tissues. The localization of brain tumor can be verified in 24 cases of 41 brain tumors-high concentration of ${ }^{32} \mathrm{P}$ can be observed in the case of metastati carcinoma, heterotopic malignant hypophyseal adenoma, astroblastoma, oligoden droglioma, craniopharyngioma and meningioma. Of these, oligodendrogioma anc craniopharyngioma forming cystic tumor have a high concentration of ${ }^{32} \mathrm{P}$ ir cystic membrane.

As to cerebral abscess, is also obtained a high concentration in an abscess membrane. But local diagnosis by this method has not much value in acoustic neurinoma, hypophyseal adenoma and intracranial hematoma. If G.M.-Tube is improved, this test may be useful to localize and to demarcate the brain tumor at operation.

\title{
7. Hormone Replacement Therapy before and after the Operation for Brain Tumor
}

\author{
Miyoshi Urabe, Takashi Tubokawa, Yoshiyuki Matsuda, \\ Yoichi OHTA, and Rokubei UCHINAMI \\ Dept. of Surgery, School of Medicine, University of Kanazawa
}

Using an adrenocortical 11-Beta-Hydroxylase inhibitor (SU-4885), the pituitary reserve was estimated in 13 of the clinical case of the tumor around the third ventricle and in 7 of the other brain tumors.

1) The SU-test revealed distinctly the disturbance of endocrinological circumstance of the brain tumor patients.

2) If the SU-test was done before the operation, the result indicated the extent of the operation of the pituitary tumor regardless of the clinical manifestations, and served for the hormone replacement therapy after the operation.

\section{Late Results of Endocrine Disorders Associated with Parasellar Tumors}

\author{
Katuhiro NiITU, Kokai NishIKAwa and Takashi TAKAHASHI \\ Dept. of Surgery, Iwate Medical College
}

Hypothalamopituitary endocrinologic functions are disturbed and diminished 
after compression or infiltration of parasellar tumors. The most important problem after radical removal of tumor is that either the diminished functions will be able to regenerate or not.

On the 2 cases of parasellar tumors operated at puberty were reported. The 1st case was 22 years old male who was diagnosed as craniopharyngioma and the tumor was radically removed 8 years ago. The 2 nd case was 21 years old female who diagnosed as optic glioma and partial resection was performed and treated by heavy radiation therapy. In both cases the endocrinologic functions which were already extremely disturbed could not reappear even after several years of complete cure of tumor.

\title{
9. Endocrinological Aspects of Postoperative Cases of Sellar Tumors
}

\author{
Tohru Uozumi, Ryoichi Nozaki, Akira Kitagawa and \\ Kenzo MatsuoKa \\ Ist Surgical Department, Medical School, Osaka University
}

We made an endoclinological study on 17 cases of the sellar tumor for which the operation was done during 5 years since 1958.

1) In 3 cases, the adrenocortical response, which was below the normal range preoperatively, returned to the normal within one year after the operation. However, the feed-back mechanism which was studied by the SU-4885 test was not normal.

2) When the sexual function was already below the normal range preoperatively, in no cases, the function did not return to the normal postoperatively, even if the adrenocortical function became normal. In those cases, a decrease of gonadotropin, a marked decrease of etiocholanolone and androsterone, amenorrhea, and no response of ovaries to exogenous gonadotropin were noted.

\section{Significances of Enzyme Activity in Neurosurgical Disease}

\author{
Tetsuro Miwa, Kozaburo Kimura and Iwao IwaKI \\ Dept. of Surgery, Tokyo Medical College
}

Recently, it has been attempted to measure enzyme activity of serum and 